\title{
Influence of Substrate Heat Treatment to the Structural Properties of Nd:YAG Thin Films Produced by Femtosecond Pulsed Laser Deposition
}

\author{
Lean L. Dasallas* \\ National Institute of Physics, University of the Philippines-Diliman, Quezon City, 1101 Philippines \\ Arriane P. Lacaba \\ National Institute of Physics, University of the Philippines-Diliman, Quezon City, 1101 Philippines, and \\ Maxim Philippines Operating Company, Gateway Business Park, General Trias Cavite, 4107 Philippines \\ Wilson O. Garcia \\ National Institute of Physics, University of the Philippines-Diliman, Quezon City, 1101 Philippines \\ (Received 6 January 2017; Accepted 27 February 2017; Published 30 March 2017)
}

\begin{abstract}
The crystallinity, surface morphology and topography of the femtosecond pulsed laser deposited Nd:YAG film under in-situ and post deposition heat treatment were examined. Heat treatment improved the crystallinity of the film with the increase in the reflecting planes shown in X-ray diffraction data. Scanning electron micrograph of the heat treated film surface indicates a small degree of melting. Surface topography of the as-deposited Nd:YAG film under AFM shows size variation to within few tens of nanometers indicating the generation of nanoparticles. Temperature dependence of the cross section area, height and surface roughness of the film was determined and explained by the volume free energy of the film. Our results demonstrate the novel use of femtosecond laser to ablate and deposit laser crystal as well as heat treatment to engineer the structural properties of the film.

[DOI: $10.1380 /$ ejssnt.2017.25]
\end{abstract}

Keywords: Laser methods; Growth; Surface structure, morphology, roughness, and topography

\section{INTRODUCTION}

Lasers with pulse duration in the nanosecond domain such as excimer and Nd:YAG lasers have been used in pulsed laser deposition (PLD) as an excitation source $[1,2]$. Thin films of different materials such as superconductor $[3,4]$ and semiconductor [5] as well as devices [6, 7] have been fabricated by this technique. Recently, there is growing interest in the use of ultrafast femtosecond (fs) pulsed lasers for ablation and deposition $[2,8]$. The fs laser pulse duration is much less than the electron-lattice interaction time as well as the electron heat conduction time $[9,10]$. Heat generated in the process does not have enough time to propagate in the target due to its small thermal diffusion length $[1,9]$. The thermal damage to the target is minimized, reducing the number of particulates deposited on the substrate $[1,9,11]$ and improving the quality of the film.

One novel application of PLD is fabrication of thin film laser crystal, in which good optical, mechanical and thermal properties are needed. One of the most popular is the neodymium doped yttrium aluminium garnet (Nd:YAG) due to its spectral and lasing characteristics [12]. Solid state laser crystals are usually produced in bulk form. $\mathrm{Nd}$ :YAG or other crystals made into thin film can take advantage of the photon confinement resulting from its reduced dimension. Confinement occurs by the repeated internal reflection due to differences in the refractive index of the crystal with respect to its surrounding. It can result in a more efficient and lower onset of laser oscillation $[13,14]$. Other innovative optoelectronic applications of thin film laser crystals include waveguide lasers [13-15] and microchip laser [16, 17].

\footnotetext{
* Corresponding author: ldasallas@nip.upd.edu.ph
}

Physical vapor deposition technique such as PLD have inherent limitations restricting the properties of the film produced [18]. As a result, heat treatment is usually employed to improve the quality of the deposited film $[4,19]$. In general, there are 2 types of heat treatment. In-situ heat treatment refers to application of heat during deposition. In post-deposition heat treatment, heat is applied to the substrate and film after the deposition process. Thin films prepared by nanosecond pulsed laser with heat treatment must consider the large sized particulates produced $[20,21]$. On the other hand, fs pulsed laser deposition results in a fewer number of particulates $[1,9,11]$, making heat treatment investigations more controllable.

In this work, we investigate the characteristics and properties of Nd:YAG thin film on Si substrate fabricated by PLD using a fs Ti:Sapphire laser as the excitation source. The films are characterized and studied using X-ray diffraction analysis, atomic force microscopy and scanning electron microscopy. The effects of post deposition and in-situ substrate heat treatment on crystallinity, surface morphology and surface topography of the deposited Nd:YAG thin film are also examined.

\section{EXPERIMENTAL}

A $500 \mathrm{~mW}$ mode-locked fs Ti:Sapphire laser (Spectra Physics Tsunami) operating at $808 \mathrm{~nm}, \approx 100$ fs pulse duration and $80 \mathrm{MHz}$ repetition rate ablates the Nd:YAG target and form the deposition flux. The target exhibits high absorption at this wavelength [12]. A rotating mount is provided for the target inside the vacuum chamber. The laser beam was focused on the target using a lens. The pressure inside the vacuum chamber was brought down using a combination of rotary pump and turbomolecular pump. The thin film was deposited on silicon (110) and (111) substrates in a wide range of deposi- 


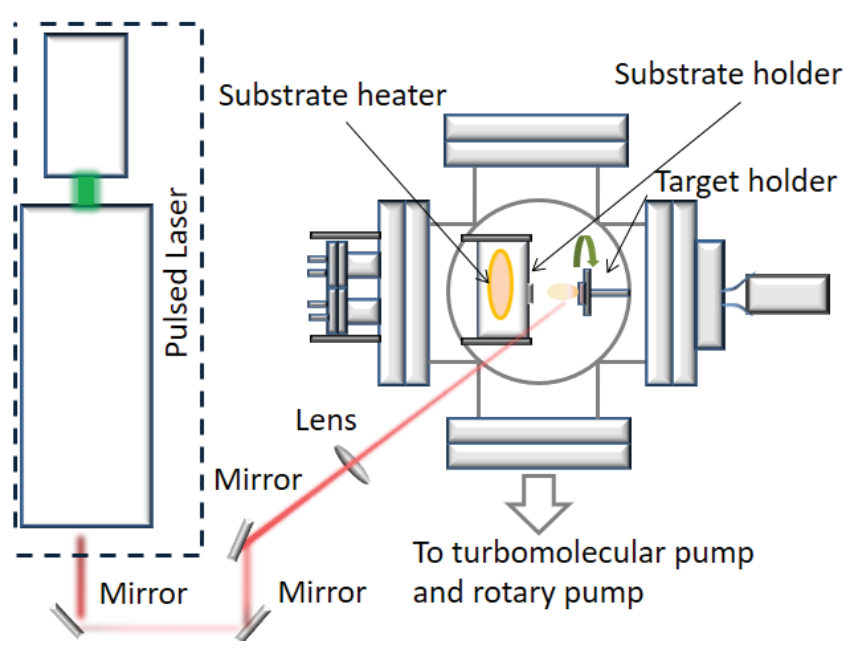

FIG. 1. Schematic diagram of the experimental set-up.

tion period ( 2 hrs- 6 hrs), deposition pressure $\left(10^{-2}\right.$ mbar$10^{-6}$ mbar) and target-to-substrate distance $(2 \mathrm{~cm}-5 \mathrm{~cm})$ with no background gas. In-situ heat treatment as well as post deposition heat treatment were implemented through radiative heating using a $1 \mathrm{~kW}$ halogen lamp located at the back of the substrate. A thermocouple sensor near the substrate monitors the temperature. The silicon substrates were processed using RCA cleaning method. The schematic diagram of the experimental set-up is shown in Fig. 1.

Surface morphology, crystallinity and surface topography of the deposited films were investigated using scanning electron microscope (Philips XL30), X-ray diffractometer (Bede D3 XRD) and atomic force microscope (NTMDT Solver Pro).

\section{RESULTS AND DISCUSSION}

Figure 2 shows the XRD spectrum of the (a) Nd:YAG target, (b) as-deposited Nd:YAG film, and (c) in-situ heat treated Nd:YAG film. The wavelength used in the X-ray diffractometer is $1.5406 \AA$ with scan step size is $0.050^{\circ}$. The as-deposited films were grown at $3 \mathrm{~cm}$ target-tosubstrate distance and $2 \mathrm{hrs}$ deposition period while the in-situ heat treated films were deposited for $3 \mathrm{hrs}$ at $2 \mathrm{~cm}$ target-to-substrate distance. Peaks corresponding to the (321) and (611) planes indicate that films have preferred crystallographic orientation on the Si substrate. Improvement in crystallinity of the in-situ heat treated films deposited at substrate temperature of $T_{s u b}=400^{\circ} \mathrm{C}$ for $3 \mathrm{hrs}$ (Fig. 2(c)) were observed with the appearance of the (422) reflecting plane. The number of reflecting planes increased with the improved mobility of the deposited particles to orient themselves. The additional energy from the heat treatment allowed atoms to adjust and relax in their corresponding lattice sites.

SEM micrograph of the as-deposited film grown for $3 \mathrm{hrs}, \approx 10^{-6}$ mbar, and $2 \mathrm{~cm}$ target-to-substrate distance is shown in Fig. 3(a). The micrograph shows that the morphological characteristics are uniform within the observation area. Voids are also observed, indicated by the
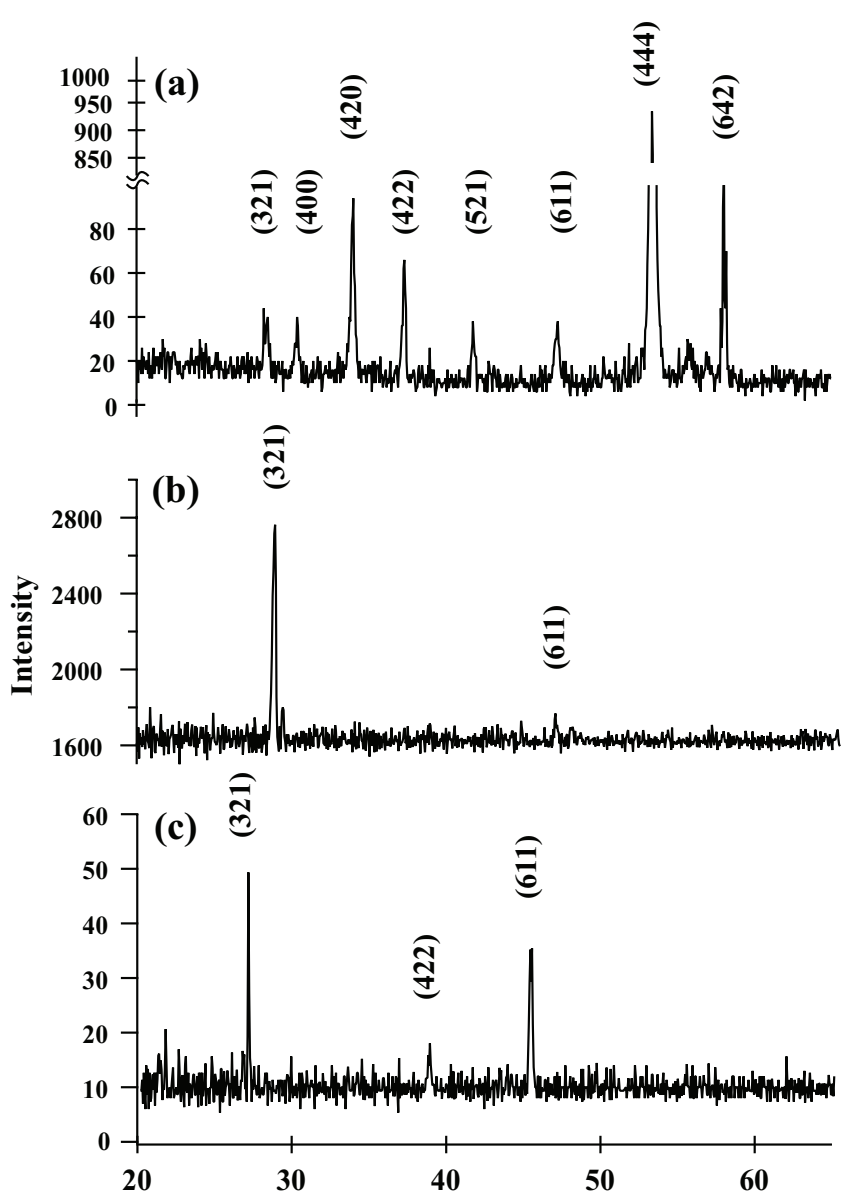

$2 \theta$ (degrees)

FIG. 2. X-ray diffraction spectrum of (a) Nd:YAG target, (b) as-deposited film and (c) in-situ heat treated film ( $T_{\text {sub }}=$ $400^{\circ} \mathrm{C}$ ). Increase in the number of diffraction planes has been observed which can be result of additional energy obtained by adatoms during heating.

arrows. Figure 3(b) and 3(c) represents the magnified surface morphology of the film deposited at substrate temperatures of $T_{\text {sub }}=400^{\circ} \mathrm{C}$ and $500^{\circ} \mathrm{C}$. The micrographs show small degree of melting in the structures due coalescence and clustering of the adatoms. The increased mobility and molecular diffusion of adatoms are gained from the energy from the radiative heater. The substrate heater is an effective method in modifying the surface properties of the film especially for waveguide applications, where smooth boundaries are important.

The surface topography of the as-deposited film fabricated at $3 \mathrm{hrs}$ deposition time, $\approx 10^{-5}$ mbar deposition pressure and $5 \mathrm{~cm}$ target-to-substrate distance are shown in Fig. 4. The image was taken using tapping mode AFM with Si tip and corrected by a linear fit. A line scan (Fig. 4-bottom) taken along the horizontal white line in the image, reveals that the variation in the height of deposited particles in the observation area to be within few tens of $\mathrm{nm}$, indicating production of nanoparticles. The short pulse duration of the femtosecond laser causes the ablation area to experience high strain rate with minimal heat transfer to the neighboring lattice site $[1,9]$. Superheated layer is not fully formed, reducing the micron-sized 
(a)

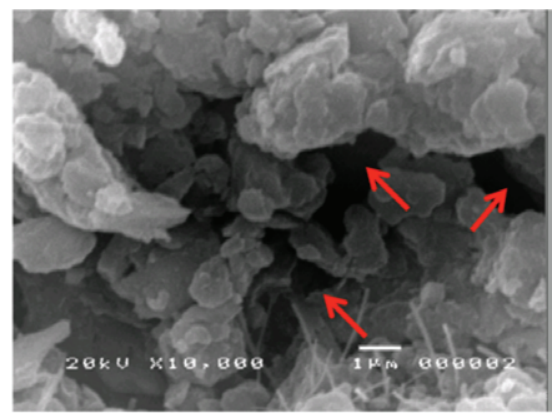

(b)

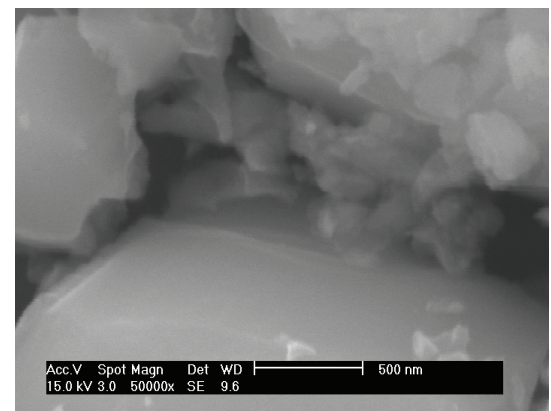

(c)

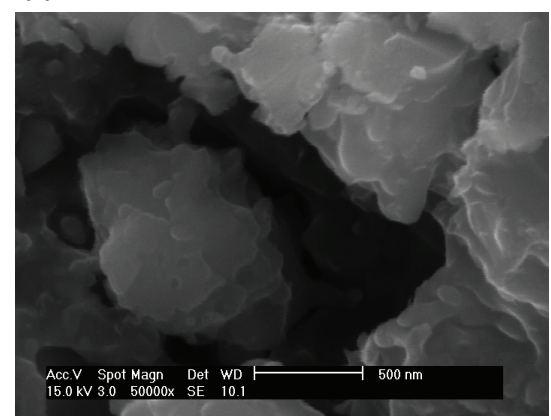

FIG. 3. SEM micrograph of the (a) as deposited film and post deposition heat treated film with (a) $T_{\text {sub }}=400^{\circ} \mathrm{C}$ and (b) $T_{\text {sub }}=500^{\circ} \mathrm{C}$. Coalescence and clustering of the deposited particles are observed.

particulates from the ejected liquid droplets [9]. However, the material can be ablated due to the fragmentation as a effect of isochoric heating decomposition in the high strain ablation site $[9,22]$ resulting to ejection of the nanoparticles that has been observed in the AFM image. This production of the nanoparticles can be utilized to investigate in detail the influence of the substrate heating effect in the deposited film.

Nd:YAG thin films were prepared with in-situ and post deposition substrate heat treatment at $3 \mathrm{hrs}$ deposition time, $\approx 10^{-6}$ mbar deposition pressure and $2 \mathrm{~cm}$ target-tosubstrate distance. The temperature was increased from room temperature to the desired substrate temperature and brought back to room temperature after 3 hrs of heat treatment.

Figures 5(a), 5(b) and 5(c) shows the atomic force micrograph of the films deposited with in-situ substrate heat treatment of temperatures $300^{\circ} \mathrm{C}, 450^{\circ} \mathrm{C}$ and $600^{\circ} \mathrm{C}$, respectively. The surface topography of the films subjected to post deposition substrate heat treatment of $400^{\circ} \mathrm{C}$, $500^{\circ} \mathrm{C}$ and $600^{\circ} \mathrm{C}$ are shown in Figs. 5(d), 5(e) and 5(f).

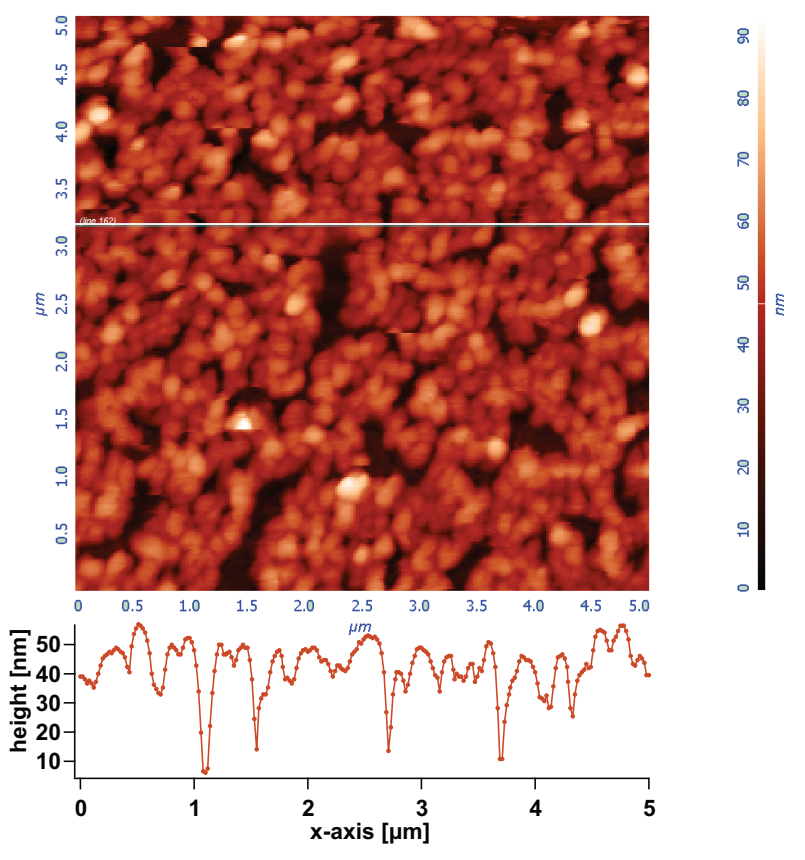

FIG. 4. Surface topography of the as-deposited film. Line profile (shown at the bottom) reveal that the variation on the height of the deposited Nd:YAG is in the order of few tens of nanometer and is distributed uniformly in the scan area.

The scan area of all the images are $10 \mu \mathrm{m} \times 10 \mu \mathrm{m}$. The images are corrected by a linear fit. Both in-situ and post deposition heat treatment of films results to decrease in the size of the deposited particles with temperature increase compared to films without heat treatment. More islands are also found in the post deposition heat treated films compared to the in-situ heat treated films. This behaviour is traced to the volume free energy per unit volume of the clustered particles given by the equation

$$
\Delta G_{V}=-\frac{k T}{\Omega} \ln \left(\frac{P}{P_{e}}\right),
$$

where $k$ is the Boltzmann's constant, $T$ the absolute temperature, $\Omega$ the atomic volume of the film atoms, $P$ the pressure of the incoming ablated atoms in the surface of the substrate and $P_{e}$ the vapour equilibrium pressure $[23,24]$. Decreasing $\Delta G_{V}$ would result to a decrease in the total free energy barrier for cluster formation that can induce nucleation and island growth $[23,24]$ on the substrate. However, an increase would lead to a monolayer growth of the film [23, 24]. Equation (1) dictates that volume free energy of the as-deposited film would depend solely on the deposition pressure. However, the temperature term becomes more significant if substrate heating effects are considered.

To compare the two substrate heating mechanism, we introduce $P=\alpha P_{0}$, in which $P_{0}$ the inherent pressure in the film and substrate when there is no deposition and $\alpha$ is a positive valued coefficient $(\alpha \geq 1)$. The repetition rate of the femtosecond laser is high $(\approx 80 \mathrm{MHz})$ so the pressure $P$ can be assumed to be quasi-continuous. We also introduce $P_{e}=\beta P_{1}$, in which $P_{1}$ is the vapour pres- 

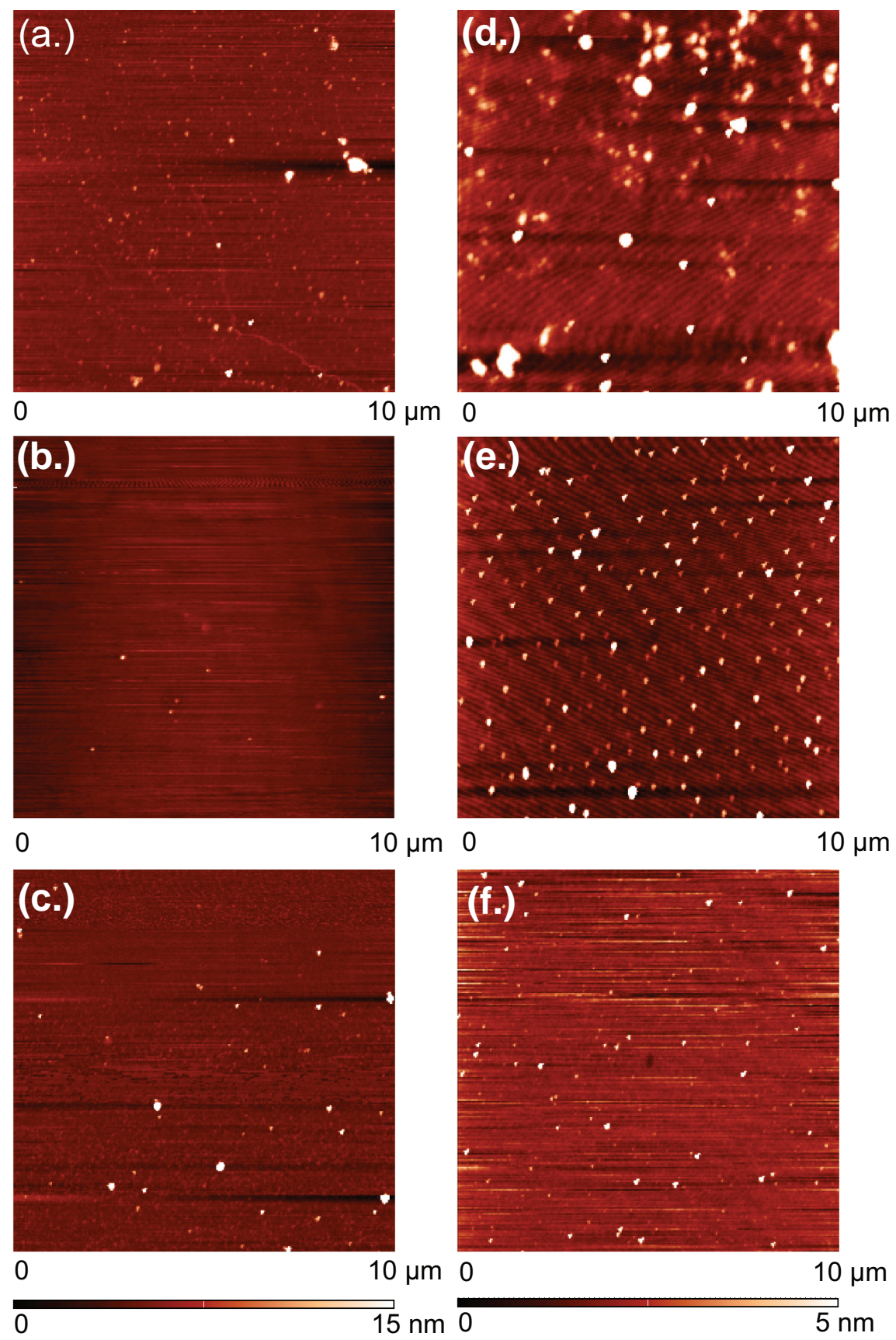

FIG. 5. Surface topography of films subjected to in-situ heat treatment with temperatures of (a) $300^{\circ} \mathrm{C}$, (b) $450^{\circ} \mathrm{C}$ and $(\mathrm{c})$ $600^{\circ} \mathrm{C}$ as well as films subjected to post deposition heat treatment with temperatures of (d) $400^{\circ} \mathrm{C}$, (e) $500^{\circ} \mathrm{C}$ and (f) $600^{\circ} \mathrm{C}$. The dimension of each image is $10 \mu \mathrm{m} \times 10 \mu \mathrm{m}$. The scale bar at the bottom of (c) indicates the height of films subjected to in-situ heat treatment and the scale bar at the bottom of (f) indicates the height of the films subjected to post deposition treatment.

sure with no substrate heat treatment and $\beta$ a positive valued coefficient $(\beta \geq 1)$.

During in-situ substrate heat treatment, the growth of the film occurs during laser ablation, so the coefficient $\alpha$ is greater than 1 . The vapour equilibrium pressure also tends to increase with substrate temperature, so the coefficient $\beta$ is also greater than 1. Equation (1) can be expanded to

$$
\Delta G_{V, \text { in }- \text { situ }}=-\frac{k T}{\Omega} \ln \left(\frac{P_{0}}{P_{1}}\right)+\frac{k T}{\Omega}(\ln \beta-\ln \alpha) .
$$

On the other hand, the growth on post deposition heat treatment is a 2 -step process. The first step involves deposition of ablated materials from the target where there is laser ablation but no heat treatment, making $\beta=1$ and $\alpha>1$. The volume free energy equation will then be

$$
\Delta G_{V, \text { post-dep } 1}=-\frac{k T}{\Omega} \ln \left(\frac{P_{0}}{P_{1}}\right)+\frac{k T}{\Omega}(-\ln \alpha) .
$$

The second step involves heat treatment but no ablation, giving $\beta>1$ and $\alpha=1$. This leads to the volume 


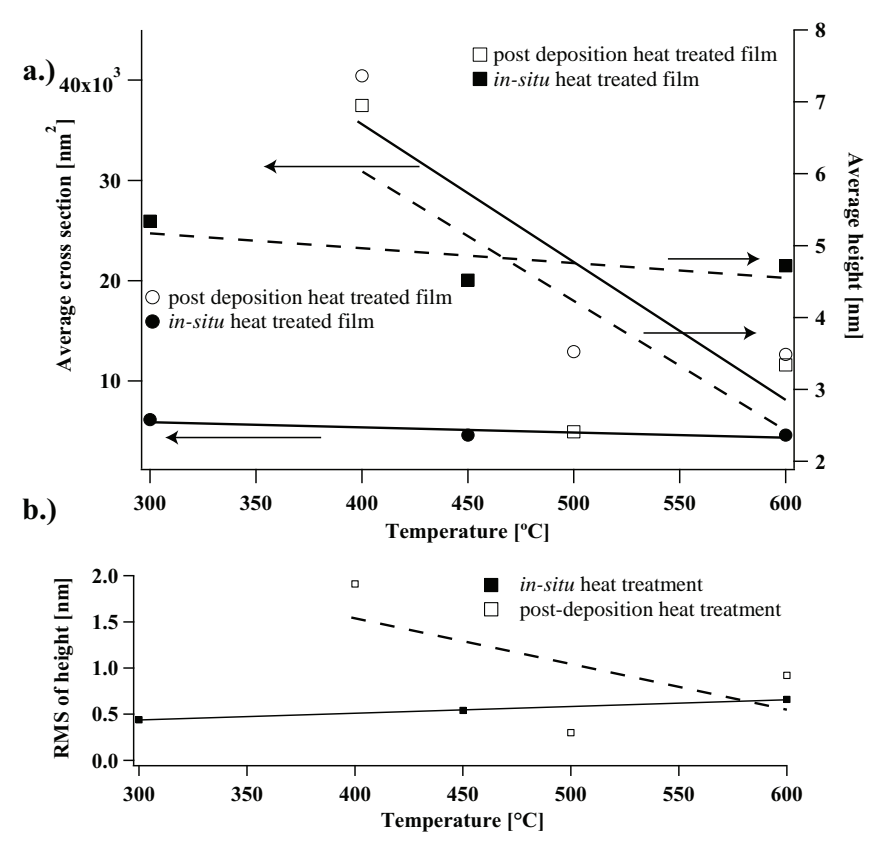

FIG. 6. (a) Temperature dependence of the average cross sectional area and average height of in-situ heat treated and post deposition heat treated Nd:YAG films and (b) root-meansquare (RMS) of the height of the in-situ heat treated and post deposition heat treated Nd:YAG films. It can be seen that insitu heat treatment and post deposition heat treatment have different effects on the average cross sectional area and the height of the deposited particles.

free energy equation

$$
\Delta G_{V, \text { post }-\mathrm{dep} 2}=-\frac{k T}{\Omega} \ln \left(\frac{P_{0}}{P_{1}}\right)+\frac{k T}{\Omega}(\ln \beta) .
$$

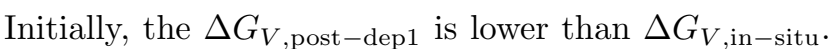
The volume free energy for nucleation is higher in the $i n$ situ heat treated films. The different values of volume free energy are manifested as more island growth in the first process of post deposition heat treatment compared with in-situ heat treatment. This behavior is seen in Fig. 5 . However, upon the application of heat treatment to the deposited films, the $\Delta G_{V \text {,post-dep2 increases and becomes }}$

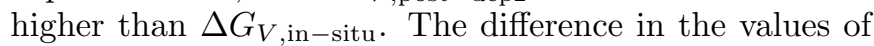
the volume free energy means that the nucleation barrier in the post deposition heat treated particles is higher than the in-situ heat treated particles.

We also investigated the changes in the average crosssectional area and the average height of the deposited particles as a function of the in-situ and post deposition substrate heat temperature. The results are shown in Fig. 6(a). The changes in the average cross sectional area and the height of the particles with respect to the substrate temperature in post deposition heat treatment is larger than the in-situ heat treated film. The difference is due to the higher substrate temperature leading to higher $\beta$. By Eqs. (2) and (4), a higher $\beta$ also lead to higher $\Delta G_{V}$. However, the increase of the volume free energy is not the same for in-situ and post deposition substrate heat treatment. The increase in $\Delta G_{V \text {,in-situ }}$ is suppressed by the pressure from the arriving atom because of laser ablation $(\alpha>1)$. Since an increase in $\Delta G_{V}$ leads to monolayer growth, the higher $\Delta G_{V \text {,post-dep2 as }}$ compared to $\Delta G_{V \text {,in-situ }}$ results in more pronounced flattening in post deposition heat treatment. The difference in $\Delta G_{V}$ results in larger decrease in the average cross section area and the average height in post deposition heat treatment compared to in-situ heat treatment. The observed flattening will also result in the decrease in the surface roughness of the deposited film as seen in Fig. 6(b), measured as the root-mean-square of the height of the deposited film for both heat treatments. Different substrate heat treatment has varied effects on the properties of the film. It is therefore an effective engineering method to improve the quality of the film.

\section{CONCLUSION}

We demonstrated the pulsed laser deposition of Nd:YAG laser crystal on Si substrate using femtosecond Ti:Sapphire laser. Nanoparticles are observed in the surface of the thin film. The crystallinity of the deposited Nd:YAG target can be improved by heat application, as manifested by the increased number of reflecting planes. Clustering and coalescence of the heat treated deposited particles are observed in the scanning electron micrograph, since the adatoms receive additional energy from the heater. The effects of the in-situ and post deposition substrate heat treatment in the deposited film have also been investigated. The results showed that flattening of the thin film is more apparent at post deposition heat treatment than in-situ substrate heat treatment due to the volume free energy of the film. Both heat treatments are found to be effective measures on engineering the surface topography and roughness of the deposited film in the microscopic level.

\section{ACKNOWLEDGMENTS}

We acknowledge the support provided by the University of the Philippines, Office of the Vice-Chancellor for Research and Development (UPD-OVCRD), the National Research Council of the Philippines (Project No. P-016) and the UP System Enhanced Creative Work and Research Grant.
[1] E. Gamaly, Theory, Experiments and Applications: Femtosecond Laser-Matter Interactions (Pan Stanford Publishing, Singapore, 2011).

[2] S.-C. Chen, et al., Nanoscale Res. Lett. 9, 280 (2014).
[3] J. C. De Vero, L. F. J. Gabayno, W. O. Garcia, and R. V. Sarmago, Physica C 470, 149 (2010).

[4] J. C. De Vero, G. R. S. Blanca, J. R. Vitug, W. O. Garcia, and R. V. Sarmago, Physica C 471, 378 (2011). 
[5] E. Fazio, A. M. Mezzasalma, G. Mondio, T. Serafino, F. Barreca, and F. Caridi, Appl. Surf. Sci. 257, 2298 (2011).

[6] B. Yang, M. Mahjouri-Samani, C. M. Rouleau, D. B. Geohegan, and K. Xiao, Phys. Chem. Chem. Phys. 18, 27067 (2016).

[7] D. Liu, X. Li, C. Shi, and Q. Liang, Mater. Lett. 188, 271 (2017).

[8] M. S. B. Darby, T. C. May-Smith, R. W. Eason, T. Donnelly, J. G. Lunney, and K. D. Rogers, Appl. Surf. Sci. 254, 3364 (2008).

[9] P. Balling and J. Schou, Rep. Prog. Phys. 76, 036502 (2013).

[10] P. V. Petkov, S. S. Dimov, R. M. Minev, and D. T. Pham, Proc. IMechE. Part B: J. Eng. Manufacture 222, 35 (2008).

[11] J. Perrière, E. Milon, W. Seiler, C. Boulmer-Leborgne, V. Craciun, O. Albert, J. C. Loulergue, and J. Etchepare, J. Appl. Phys. 91, 690 (2002).

[12] W. Koechner and M. Bass, Solid State lasers: A graduate text (Springer, New York, 2003).

[13] M. Ezaki and M. Obara, Appl. Phys. Lett. 69, 2977 (1996).
[14] H. Kumagai, K. Adachi, M. Ezaki, K. Toyoda, and M. Obara, Appl. Surf. Sci. 109-110, 528 (1997).

[15] R. W. Eason, S. J. Barrington, C. Grivas, T. C. MaySmith, and D. P. Shepherd, in Pulsed laser deposition of thin films: Application-Led growth of functional materials, 2nd ed., Ed. R. Eason (John Wiley and Sons, New Jersey, 2007), p. 385.

[16] J. J. Zayhowski, Opt. Mater. 11, 255 (1999).

[17] J. J. Zayhowski, J. Alloys Compd. 303-304, 393 (2000).

[18] P. R. Willmott and J. R. Huber, Rev. Mod. Phys. 72, 315 (2000).

[19] K. Punitha, R. Sivakumar, C. Sanjeeviraja, and V. Ganesan, Appl. Surf. Sci. 344, 89 (2015).

[20] M. N. R. Ashfold, F. Claeyssens, G. M. Fuge, and S. J. Henley, Chem. Soc. Rev. 33, 23 (2004).

[21] C. Liu, X. L. Mao, S. S. Mao, X. Zeng, R. Greif, and R. E. Russo, Anal. Chem. 76, 379 (2004).

[22] P. Lorazo, L. J. Lewis, and M. Meunier, Phys. Rev. Lett. 91, 225502 (2003).

[23] J. E. Greene, in Multicomponent and Multilayered Thin Films for Advanced Microtechnologies, Eds. O. Auciello and J. Engemann (Kluwer Academic, Netherlands, 1993).

[24] P. R. Willmott, Prog. Surf. Sci. 76, 163 (2004). 